\title{
The prevention of hospital/clinic attendance, or admission of home enteral nutrition tube-feeding patients living in the Avon area
}

\author{
D. Reeves ${ }^{1}$, L. Harrison ${ }^{1}$, S. Croucher ${ }^{1}$, T. McQueen ${ }^{1}$, L. Martin ${ }^{2}$ and C. Smyth ${ }^{2}$ \\ ${ }^{1}$ Clinical Nurse Advisors, Fresenius Kabi Ltd, WA7 1NT and ${ }^{2}$ Home Management Services, United Bristol Hospital Trust, \\ BS2 $8 H W$
}

The purpose of this audit was to demonstrate the effectiveness of the Fresenius Kabi Homecare Nursing Team in preventing homeenterally-fed patients attending or being admitted to hospital/clinic.

The Nursing Service in Avon supports 440 patients with two adult and two paediatric full time registered nurses. In 2004 and 2005 the Nursing Service was audited and information collated by Caroline Smyth (CNS Nutrition, United Bristol Hospital Trust) with the results showing that over $50 \%$ of Nurse Advisor visits/trainings were planned thus demonstrating a proactive service; $23 \%$ of Nurse Advisor visits were urgent, demonstrating a reactive service and $15 \%$ percent of Nurse Advisor visits were annual reviews; furthermore $10 \%$ of nursing activity was related to Nurse Advisors providing telephone advice.

A total of 569 patient visits were audited over a six-month period (July-December 2006) and nursing intervention recorded on an audit record sheet. The patient visits included tube-fed patients who where unable to receive their feed and required intervention.

The audit record sheet incorporated the following issues: (1) blocked feeding tube; (2) feeding tube replacement; (3) feeding pump failure/user error; (4) unsuitable ancillaries; (5) Urgent feeding regimen change; (6) carer unable to provide feed; (7) other clinical advice/ action or liaison (Table).

During this period 184 hospital/clinic attendances or admissions were prevented (ninety adults and ninety-four paediatrics).

\begin{tabular}{lrrr}
\hline Presenting issues $(n)$ & Total & Adult & Paediatric \\
\hline Blocked tubes & 15 & 14 & 1 \\
Tube replacements & 124 & 49 & 75 \\
Pump failure & 15 & 10 & 5 \\
Unsuitable ancillaries & 22 & 10 & 12 \\
Urgent training & 3 & 3 & 0 \\
Other clinical advice & 5 & 4 & 1 \\
Visit type & & & \\
Total number of patient visits $(n)$ & 569 & 325 & 244 \\
Prevented admissions/attendance (PA; $n)$ & 184 & 90 & 94 \\
Visits that were PA $(\%)$ & 32 & 28 & 38 \\
\hline
\end{tabular}

The provision of a specialist Home Enteral Tube Feeding (HEFT) Nursing Service in the Avon area is effective in reducing the number of home-enterally-fed patients attending or being admitted to hospital/clinic. This audit also demonstrates potential financial savings for Primary Care Trusts (PCT) and hospital Trusts. Ongoing data collection and analysis is being produced with PCT-specific results demonstrating cost savings. 